03

\title{
A novel approach to realize of all optical frequency encoded dibit based XOR and XNOR logic gates using optical switches with simulated verification*
}

\author{
(C) B. Ghosh ${ }^{1}$, S. Hazra ${ }^{1}$, N. Haldar ${ }^{1}$, D. Roy ${ }^{1}$, S.N. Patra ${ }^{2}$, J. Swarnakar ${ }^{3}$, P.P. Sarkar ${ }^{1, \uparrow, ~ S . ~ M u k h o p a d h y a y ~}{ }^{4}$
}

${ }^{1}$ Department of Electronics and Communication Engineering, University Institute of Technology, The University of Burdwan, Burdwan 713104 West Bengal, India

${ }^{2}$ Department of Instrumentation Science, Jadavpur University, Kolkata 700032, West Bengal, India

${ }^{3}$ Department of ECE, North Eastern Hill University, Shillong, Meghalaya 793022 India

${ }^{4}$ Department of Physics, The University of Burdwan, Burdwan

713104 West Bengal, India

I e-mail: pratim34@gmail.com

Received April 04, 2017

Since last few decades optics has already proved its strong potentiality for conducting parallel logic, arithmetic and algebraic operations due to its super-fast speed in communication and computation. So many different logical and sequential operations using all optical frequency encoding technique have been proposed by several authors. Here, we have keened out all optical dibit representation technique, which has the advantages of high speed operation as well as reducing the bit error problem. Exploiting this phenomenon, we have proposed all optical frequency encoded dibit based XOR and XNOR logic gates using the optical switches like add/drop multiplexer (ADM) and reflected semiconductor optical amplifier (RSOA). Also the operations of these gates have been verified through proper simulation using MATLAB (R2008a).

DOI: $10.21883 /$ OS.2018.03.45655.82-17

* Полный текст статьи опубликован в английской версии журнала. 